\title{
Comparative Analysis of Actual and Mental Movement Times in Two Graphic Tasks
}

\author{
Jean Decety and François Michel
}

Laboratoire de Neuropsychologie Expérimentale. Inserm U94, Bron 69500. France

\begin{abstract}
This study compared the temporal organization of graphic movements executed either actually or mentally. Six subjects had to perform two tasks, writing a sentence and drawing a cube, either as a real performance or as an imagined one, with either the right or the left hand, and with either a small or a large tracing amplitude. In the same subject, for the same hand, mental and actual movement times were both very stable and very close from trial to trial regardless of the tracing amplitude. Thus, mental movements mimic closely real movements in their temporal organization and are likcly to involve the same planning program. 1989 Academic Press. Inc.
\end{abstract}

\section{INTRODUCTION}

Most of the experimental work on mental imagery has focused on visual imagery. By comparison, motor imagery, i.e., the mental simulation of a voluntary movement on the basis of visuo-spatial and kinesthetic memorized patterns, has drawn little attention among cognitive psychologists. This is despite the fact that motor imagery (so-called mental practice) is often used by people practicing sports at a high level, such as in skiing, athletism, and diving. Indeed many experiments have shown that mental rehearsal may have positive effects on motor skill learning as well as on motor performance (Hall \& Goss, 1985).

Visual imagery studies have led several authors to accept, as a working hypothesis, a functional analogy in the information processing between visual perception and visual imagery (Finke, 1986; Finke \& Kurtzman, 1981; Kosslyn, 1978, 1984). According to this model, visual imagery and visual perception would, to some extent, activate the same cognitive processes and this could explain the interferences observed in subjective experiments (Farah, 1984). Moreover, both visual perception and visual

Acknowledgments are given to Dr. Denis Pelisson and Dr. Claude Prablanc for their useful help conducting the experiment. The authors thank Pr. R. G. Marteniuk (University of Waterloo) for his helpful comments. 
imagery may involve some processes in the same neural loci. Indeed, in cases of posterior brain lesions, both imagery and perception may be equally impaired (Basso, Bisiach, \& Luzatti, 1980; Farah, 1985; Levine, Warach, \& Farah, 1985). In addition, visual evoked potentials studies have also shown that imagery may influence perceptual processes (Farah, Peronnet, Gonon, \& Giard, 1988). Finally, blood flow studies have shown an increase of the regional cerebral blood flow ( $\mathrm{rCBF}$ ) in the superior occipital and in the posterior parietal cortex during visual imagery tasks (Goldenberg, Podreka, Steiner, \& Williams, 1987; Roland, Eriksson, Stone-Elander, \& Widen, 1987). During motor imagery, rCBF increases have been measured in the prefrontal cortex and in the supplementary motor areas (Ingvar and Philipson, 1977, Roland \& Friberg, 1985; Roland, Larsen, Lassen, \& Skinhoj, 1980) as well as in the cerebellum (Decety, Philippon, \& Ingvar, 1988).

According to the functional analogy model, a purely mental execution of a complex movement, such as writing or drawing, should entail internal information processes similar to those used when the movement is actually performed. This would imply that the timing and the duration of mental movements should mimic the timing and duration of real movements. A contrary hypothesis would be that in the absence of control by feedback, movements mentally performed could have a different timing pattern than actual movements. It is already known that the temporal pattern of speech is not affected by the absence of feedback control since implicit and explicit speech have virtually identical rates (Landauer, 1962). Conversely, mentally generating a visual pattern of alphabet letters takes more time than uttering them (Weber \& Castelman, 1970). This shows that visual imagery and motor imagery require quite different processes.

In order to investigate motor imagery and not, as far as possible, visual imagery, we have chosen to study handwriting (a sentence) and drawing (a cubc) because both tasks present some advantages. First, the temporal patterns of handwriting are well described (Hollerbach, 1981; Michel, 1971, 1976; Viviani \& Terzuelo, 1980). Second, these tasks are essentially accomplished by distal portions of limbs and can be attributed mostly to contralateral hemisphere motor control (Brinkman and Kuypers, 1973). Third, it should be possible to compare right and left hemisphere processing since handwriting a sentence is clearly a left hemisphere processing task while drawing a cube is supposed to require more the participation of right hemisphere visuo-spatial ability. This assumption is based on the fact that the functional specialization of the cerebral hemispheres is a prominent contributor to hand differences in performance (Todor and Smiley, 1985).

Regarding handwriting, it is well established that the time taken to write a word is extremely stable if the subjects write in a natural way. 
Moreover, this overall time does not vary with the size of the tracing (Michel, 1971). Indeed when subjects write larger, they also write faster: that is, the absolute velocity of the pen's tip on the paper increases in proportion to the tracing's amplitude. While the absolute velocity along the trajectory varies, its temporal profile remains roughly independent of the size. One can take the example of a subject writing the word "eagle." One will observe that the subject writes the down stroke of "g" at approximately the same time after the start, whatever the size of his tracing. However, when the nondominant hand is used, the movements will be slower and more variable.

One may assume that in motor imagery, the motor program, once initiated, will obviously not be influenced by peripheral feedback and will run its full course without sensory intervention. In such an outflow process, can one find those features which characterize actual graphic movements involving possible control by afferences? Is there a manual dominance in motor imagery? Are the mental movement times stable across trials? Is the temporal structure similar in mental and actual motor sequences? Are mental movement times influenced by the imagined size of tracing? In short, can we expect to find an isochronism which would suggest that mental activity is in some sense isomorphic to physical activity?

\section{METHOD}

To answer these above questions, a four factor experimental paradigm was adopted in order to measure and compare:

-actual movement times and mental movement times,

-movements executed with the right and the left hand,

-small or large amplitude tracings,

-two tasks, writing and drawing.

Overall, there were $2 * 8$ different conditions for each subject (Fig. 1).

\section{Subjects}

Six graduate students (three males and three females) were selected and participated in this experiment. They had been classified as vivid imagers according to their scores in visual and kinesthetic imagery assessed by self-report measures with Sheehan's questionnaire (1967). All were strongly right handed according to the Edinburg Handedness Inventory $(L Q>+85$ ). Their age ranged between 20 and 26 years.

\section{Experimental Apparatus}

Movements times were recorded by an experimental apparatus described by Prablanc, Echallier, and Jeannerod (1979) and modified for the present study as shown in Fig. 2.

This experimental apparatus, placed in an isolated room, consisted of three parallel panels $(a, b, c)$ :

(a) A conducting table which detected the contact of a metallic thimble fixed on the subject's forefinger, detecting the onset and end of the actual graphic task. 


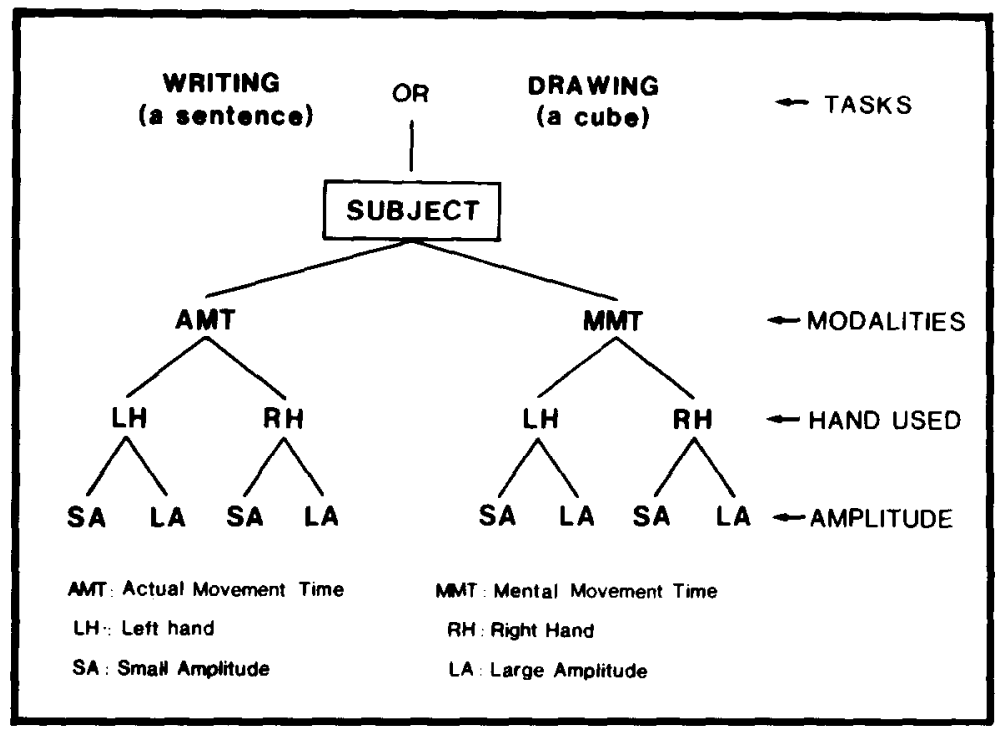

Fig. 1. Experimental paradigm.

(b) Above the table, a semi-reflecting mirror allowing the subject to see the virtual image of two white circles projected on the table $(6$ and $30 \mathrm{~cm}$ in diameter, respectively) while preventing him from seeing his hand.

(c) On the top of the apparatus, the two concentric circles with a light emitting diode (LED) in their center which was used as a fixation point for gaze direction recorded by a DC electro-oculographic (EOG) technique.

The same apparatus was used to measure the mental movement time except that the thimble was replaced by a key (g) which subjects depressed until the end of the task. This key turned the LED off, triggered EOG recording, and detected onset at the end of the trial.

Gaze position was monitored by continuous EOG recording through surface electrodes

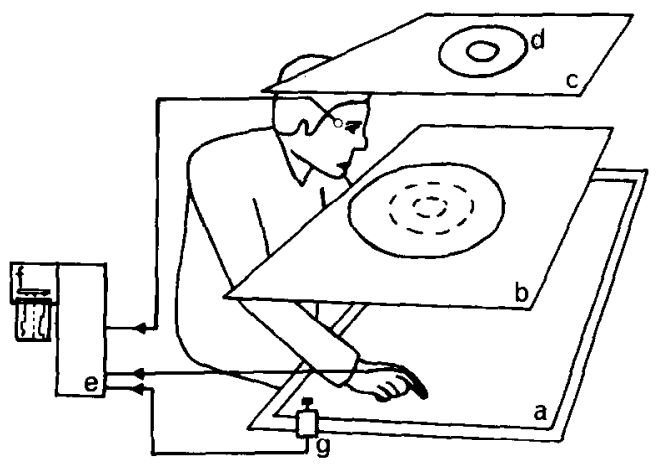

FIG. 2. Experimental apparatus for measuring actual and mental movement times. 
located near each orbit in order to obtain an objective index during the imagery task. EOG recording detected small amplitude saccades during progression along the writing line and larger amplitude saccades when subjects changed from the first to the second line.

All signals (EOG, thimble contact, key pressure) were recorded by a polygraphic system (e) with paper running at $25 \mathrm{~mm} / \mathrm{sec}$ allowing a $40-\mathrm{msec}$ resolution for time measures.

\section{Procedure}

After becoming familiar with the experimental testing. subjects were told that the experiment would involve two graphic tasks: (1) writing on two superimposed lines a sentence which is the beginning of a fable well known to all French pupils: "Maitre corbeau sur un arbre perché," "Tenait en son bec un fromage;" (2) drawing a Necker's cube with 12 lines. Subjects were told that two modalities would be required during the experiment. actual and mental.

In the actual modality, subjects were asked to perform the task with the right or the left hand using the thimble within one or the other of the two circles according to the instructions given at the beginning of each trial hy the experimentor.

In the mental modality subjects were asked to attempt to "feel" themselves making the gesture with the right or the left hand within an imagined circle. The size of the imagined circle was given by one of the real circles which appeared on the reflecting mirror for a brief period $(2 \mathrm{sec})$ before each trial. They had to maintain a key depressed until the end of the trial with the hand opposite the one "mentally used." Subjects were required not to make any movements with their imaged hand and to clench their fist.

The reason for depressing the key with the hand other than the one "mentally used" was to avoid efferent information which would have coincided with the start and the end of the task and could have given rise to proprioceptive feedback.

No information was given about the velocity of the gestures. Informal questioning at the end of the experiment indicated that none of the subjects were aware of the purposes of the study. None of the subjects reported the use of a silent speech strategy (i.e., talking to himself silently).

Each trial was preceded by verbal instructions given by the experimentor through a microphone always indicating the same order: task, modality, hand, circle.

The experimentor was outside the isolated room where the subjects were seated and never had direct contact with them during the experiment. Several practice trials preceded the experiment. Then 10 trials in each of the eight conditions and in both tasks were performed. Conditions for each task (see Fig. 1) were randomized in order to avoid a block effect, as was the order of the trials between each different subject.

A postexperimental questionnaire (Denis \& Carfantan, 1985) was administered in order to check the subject knowledge about mental imagery.

\section{RESULTS}

In order to make a clear data examination, the tasks are separately presented.

\section{Writing Task}

Mean movement times according to each condition in the writing task appear in Fig. 3. Intra-subject movement time variability within each condition was very small. Indeed the means of variation coefficients in the six subjects were: actual right hand, $4.8 \%$; mental right hand, $5.6 \%$; actual left hand, $6.5 \%$; mental left hand, $7.2 \%$. These means were not significantly different from one another $(p>0.05)$. 


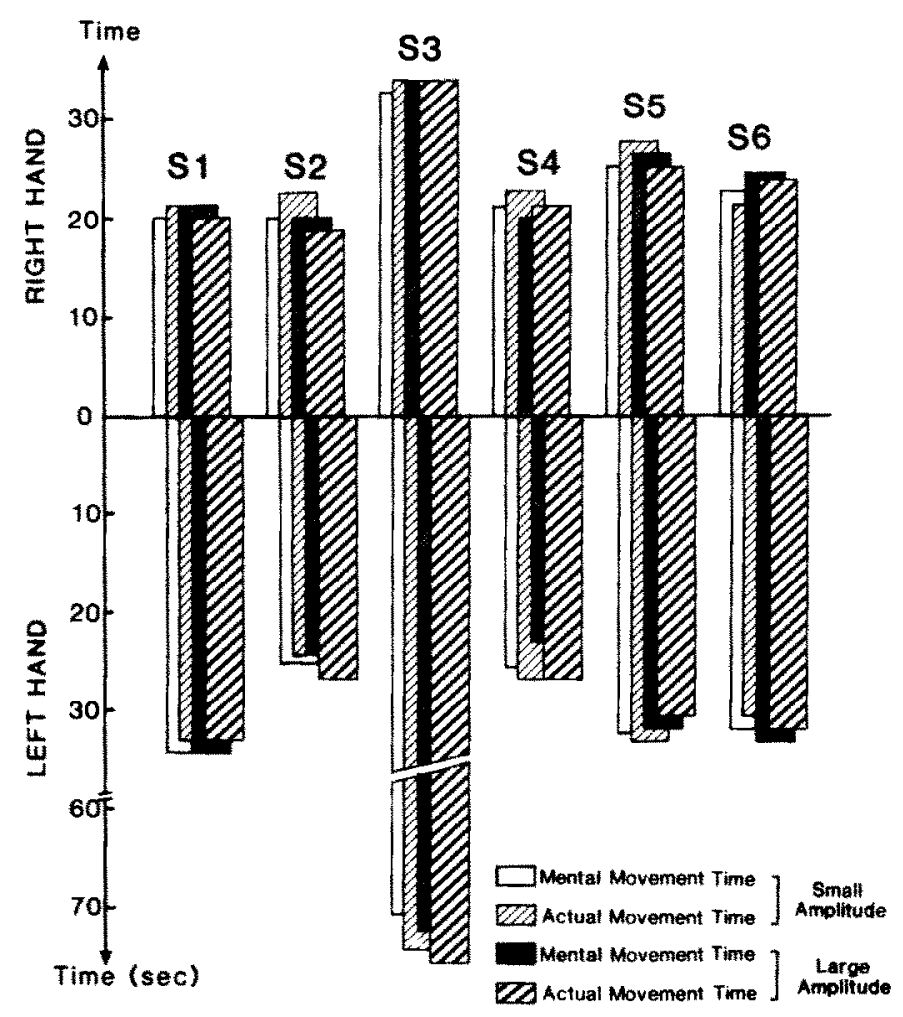

FIG. 3. Writing task: mean movement times according to actual/mental modalities, right/left hands, and small/large amplitudes for each of the six subjects.

Intra-subject analysis of movement times by three-way ANOVA revealed no significant effect for the modality, $F(1,9)$ ranged between 0.50 and $4.46, p>0.05$; no significant effect for the amplitude, $F(1,9)$ ranged between 0.27 and $5.03, p>0.05$ except for subject $2, F(1,9)=7.4$, $p<0.05$; a highly significant effect for the hand, $F(1,9)$ ranged between 523.2 and $823.5, p<0.001$; no interaction between hand and modality, $F(1,9)$ ranged between 0.06 and $5.4, p>0.5$; no interaction between modality and amplitude, $F(1,9)$ ranged between 0.27 and $1.25, p>0.5$; no interaction between hand and amplitude, $p>0.5$.

\section{Drawing Task}

Mean movement times according to each condition in the drawing task appear in Fig. 4. Again the intra-subject variability within each condition was very small since mean coefficients of variation in the six subjects were: actual right hand, $4 \%$; mental right hand, $6.6 \%$; actual left hand, $4 \%$; and mental left hand, $6.2 \%$. These means were not significantly different. 
Intra-subject analysis of movement times by three-way ANOVA revealed no significant effect for the modality, $F(1,9)=1.7$ to $4.5, p>$ 0.5 ; no significant effect for the amplitude, $F(1,9)=0.35$ to $2.4, p>$ 0.5 ; no significant effect for the hand for five subjects, $F(1,9)=0.48$ to $4.47, p>0.05$, but a significant effect for subject $5, F(1,9)=19.07$, $p<0.05$. No interactions approached significance.

A four-way within-subject ANOVA was carried out in order to determine the significant interactions. The four factors were the task, the modality, the hand, and the amplitude. Results confirmed for all subjects: a hand effect, $F(1,9)$ ranged between 12.15 and 535.51, $p<0.01$; no effect of the modalities, $F(1,9)$ ranged between 0.61 and $4.12, p>0.05$; no effect of the amplitude, $F(1,9)$ ranged between 1.14 and $5.10, p>$ 0.05 , except for subject $2, F(1,9)=7.48, p>.05$; a strong effect of the task, $F(1,9)$ ranged between 1920.03 and $11,054.28, p<0.001$; a significant interaction between the hand and the task, $F(1,9)$ ranged between 221.27 and 914.12, $p<0.001$. This can be attributed to the contribution of cerebral specialization in cognition interacting with hand motoric capability in the explanation of hand differences found in the writing task.

In order to determine if the absence of modality effect (actual vs mental) was homogeneously distributed within subjects an additional statistical control was computed within each task for each hand. Paired $t$ tests confirmed that there was no statistical differences between actual and mental movement times, $T(18)$ ranged between 0.52 and $1.53, p>$ 0.10 .

\section{Eyes Movements}

Although eye movement recording had not been formally analyzed, two observations were systematically made in both actual and mental modalities: eye movement amplitude was a function of task amplitude, and a high amplitude saccade systematically occurred when subjects came leftward for the second line. In several trials, outside the main experiment, subjects were stopped during mental or actual task at different times. It was observed that at a given time subjects often reached the same letter.

This showed that, in writing movements, the temporal patterns in both mental and actual conditions are similar. Even subjects interrupted while they were mentally writing often precisely reported which stroke inside the letter they were executing and reported, for instance: "I was just going to put the point on the i .., or "a horizontal bar on the t..." This proved that subjects were really imagining their hand writing letters and not just spelling them.

The postexperimental questionnaire indicated that subjects' knowledge about mental imagery properties was very poor. Hence, one can hardly 


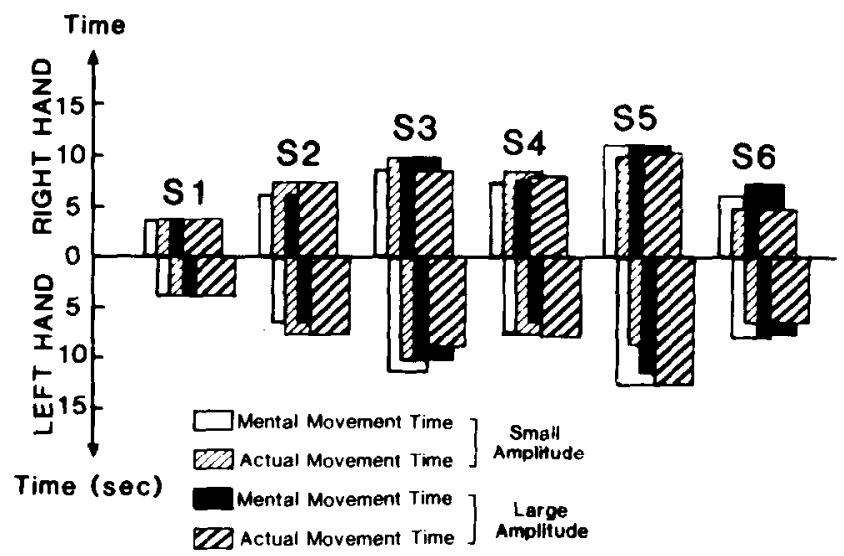

FIG. 4. Drawing task: mean movement times according to actual/mental modalities, right/left hands, and small/large amplitudes for each of the six subjects.

think that they could bias the experiment results on the basis of their knowledge.

\section{DISCUSSION}

This experiment studied motor imagery as a special cognitive process, disregarding the inter-individual variability. Accordingly the sample of subjects was limited to six "high-imagers," but additional records with other normal and neurologically impaired subjects has since confirmed the validity of the present observations (Decety \& Michel, 1987).

In the past, chronometric analysis has proven to be an objective tool for assessing mental events (Posner, 1983). The present experiment showed that chronometric assessment of mentally executed movements could also yield valuable information.

Movement times appeared very stable from trial to trial in the same subject in both modalities (actual and mental). The time required to execute a real graphic gesture and the time to execute the same gesture mentally are similar. One could have supposed that a mental movement performed without constraints (no mechanical inertia, no peripherical feedback) could be achieved more rapidly. This proved not to be the case. Nevertheless, one cannot reject the possibility of proprioceptive feedback during the imaginal condition because no electromyographic (EMG) recordings were made, although any gross muscular movements were prevented by instruction. Furthermore, some EMG studies have shown that subliminal muscular activity can be observed during motor imagery (e.g., Wehner, Vogt, \& Stadler, 1984). Therefore, this internal feedback on higher levels within the brain could possibly contribute to movement control. The question arises whether one should consider the 
peripheral myoactivity as an "effect mechanism" that is transcoding cognitive processes into an efferent signal, or a "cause mechanism." The transfer experiments of Kohl and Roenker (1983) demonstrated that motor imagery effects on muscles are different from those actually practiced. Thus their data suggest an outflow explanation of motor imagery and lead us to consider the possible peripheral EMG activity more as an effect mechanism. Another piece of evidence supporting centralistic processing of motor imagery is given by clinical observation of tetraplegic patients' ${ }^{\prime}$ which shows the same timing characteristics in mentally executed hand movements, i.e., left-right differences, little variability across trials.

The mental left hand was found to be slower than the mental right hand in the same proportion to actual left hand and actual right hand. However, while this was significant for all six subjects for the writing task, it was just a tendency for the drawing task (significant for one subject). Yet, drawing a Necker's cube is a particuliar task which requires less graphic skill than does writing and is also supposed to be more controlled by the right hemisphere. This suggest that the hands, in both mental and actual modalities, are stressed by two different factors, the hemispheric specialization of the task and the manual proficiency.

Movement times were not lengthened by increase in writing or drawing size. It has already been demonstrated that an increase in handwriting size does not automatically induce an increase in movement time; that is, handwriting movements are roughly isochronic regardless of amplitude tracing (Michel, 1971, 1976). According to the present experiment this holds also for mental writing. Durations of the mental and actual movements were similar because the temporal velocity profiles were maintained despite change in writing size, thanks to acceleration variations.

This last point is of great importance when one discusses cognitive processes underlying mental imagery. Naive subjects naturally believe that writing larger should take more time; i.e., they are not aware of the isochronism feature of handwriting. If during the mental imagery task the subjects would have tried to cope with their tacit knowledge, particularly their visual knowledge (writing larger "should" require more time), they would have biased the movement times in the direction of lengthening. This observation may be opposed to Pylyshyn's criticism (1981) against mental imagery experimental paradigms.

What sort of "image" our subjects are experiencing is a matter of debate. Motor imagery most likely involves both visual and kinesthetic internal representations. One could suppose that the subjects visualized

' Decety, J., Boisson, D., \& Michel, F. 1989. Brain injuries effects on cognitive-motor processes: An experimental neuropsychological study of motor imagery. Manuscript in preparation. 
words one after the other as whole gestalts, or letter by letter in a serial manner. In this case they focused on a visual-like experience. One could also suppose that they adopted a verbal strategy, spelling letter by letter the word they were imagining. However, this verbal strategy can certainly not be involved in cube drawing and can hardly explain the difference between right and left hands. In fact during this experiment the subjects "felt" themselves writing or drawing stroke by stroke rather than visualizing an image. The fact that size variations of imagined handwriting did not change movement times suggests that the subjects carried on a sensory motor-like experience rather than a visual experience.

These preliminary results suggest that both actual and mental movements may be governed by the same motor program regarding at least amplitude and time constraints. Finally, the study of timing of mentally exccuted movements may be a mean to focus on the outflow component of motor imagery.

\section{REFERENCES}

Basso, A., Bisiach, E., \& Luzatti, C. 1980. Loss of mental imagery: A case study. Neuropsychologia, 18, 435-442.

Brinkman, J., \& Kuypers, H. 1973. Cerebral control of contralateral and ipsilateral arm, hand, and fingers movements in the split-brain Rhesus monkey. Brain, 96, 653-674.

Decety, J., \& Michel, F. 1987. L'image mentale motrice. Second symposium national de rééducation fonctionelle, Concilia Médica. 1, 6. 175-181.

Decety, J., Philippon, B., \& Ingvar, D. H. 1988. rCBF landscapes during motor performance and motor ideation of a graphic gesture. European Archives of Psychiatry and Neurological Sciences, 238, 33-38.

Denis, M., \& Carfantan, M. 1985. People's knowledge about images. Cognition, 20, 4960.

Farah, M. J. 1984. The neurological basis of mental imagery: A componential analysis. Cognition, 18, 245-272.

Farah, M. J., Peronnet, F., Gonon, M. A., Giard, M. H. 1988. Electrophysiological evidence for a shared representational medium for visual images and visual percepts. Journal of Experimental Psychology: General, 117, 248-257.

Finke, R. A. 1986. Mental imagery and the visual system. Scientific American, 3, 76-83.

Finke, R. A., \& Kurtzman, M. S. 1981. Mapping the visual field in mental imagery. Journal of Experimental Psychology, 4, 110, 501-517.

Goldenberg, G., Podreka, I., Steiner, M., \& Willmes, K. 1987. Patterns of rcgional ccrcbral blood flow related to memorizing of high and low imagery words: An emission computer tomography study. Neuropsychologia, 25, 3, 473-485.

Hall, C., \& Goss, S. 1985. Imagery research in motor learning. In D. Goodman, R. B. Wilberg, and I. M. Franks (Eds.), Differing perspectives in motor learning, memory, and control. Amsterdam: Elsevier Science Publishers.

Hollerbach, J. M. 1981. An oscillation theory of handwriting. Biological Cybernetics, 39, 139-156.

Ingvar, D. H., \& Philipson, L. 1977. Distribution of cerebral blood flow in the dominant hemisphere during motor ideation and motor performance. Annals of Neurology, 2, 230-237.

Kohl, R. M., \& Roenker, D. L. 1983. Mechanism involvement during skill imagery. Journal of Motor Behavior, 15, 2, 179-190. 
Kosslyn, S. M. 1978. Visual images preserve metric spatial information. Journal of Experimental Psychology H.P.P., 4, 1, 47-60.

Kosslyn, S. M. 1981. The medium and the message in mental imagery: A theory. Psychological Review, 88, 46-65.

Kosslyn, S. M. 1984. The structure of the visual imagery processing system: Evidence from functional dissociations. Attention and Performance XI, Eugene, Oregon.

Landauer, T. 1962. Rate of implicit speech. Perceptual and Motor Skills, 15, 646.

Levine, D. M., Warach, J., \& Farah, M. 1985. Two visual system in mental imagery. Neurology, 35, 7, 1018-1080.

Miclıel, F. 1971. Elude experinentale du geste graphique. Neuropsychologia, 9, 1-13.

Michel, F. 1976. Methode de receuil et d'exploitation quantitative de paramètres temporospatiaux du geste graphique. Cahiers de Psychologie, 19, 39-53.

Posner. M. I. 1983. Chronometric exploration of mind. New York: Oxford University Press.

Prablanc, C., Echallier, J. F., \& Jeannerod, M. 1979. Optimal response of eye and motor systems in pointing at visual target. Biological Cybernetics, 35, 113-124.

Pylyshyn, Z. W. 1981. The imagery debate: Analogue media versus tacit knowledge. Psychological Review, 88, 1, 16-45.

Roland, P. E., Eriksson, L., Stone-Elander, N., \& Widen, L. 1987. Does mental activity change the oxidative metabolism of the brain? Journal of Neturoscicnces, 7, 8, 23732389.

Roland, P. E., \& Friberg. L. 1985. Localization of cortical areas activated by thinking. Journal of Neurophysiology, 53, 5, 1219-1243.

Roland, P. E., Larsen, B., Lassen, N. A., \& Skinhoj, E. 1980. Supplementary motor area and other cortical areas in organization of voluntary movement in man. Journal of Neurophysiology, 43, 1, 118-136.

Sheehan, P. W. 1967. A shortened form of Betts' questionnaire upon mental imagery. Journal of Clinical Psychology, 23, 386-389.

Todor, J. I., \& Smiley, A. L. 1985. Performance differences between the hands: Implications for studying disruption to limb praxis. In E. A. Roy (Ed.), Neuropsychological studies of apraxia. Amsterdam: Elsevier Science Publishers, B.V. (North-Holland).

Viviani, P., \& Terzuelo, C. 1980. Space-time invariance in learned motor skills. In G. E. Stelmach and J. Requin (Eds.), Tutorials in Motor Behavior. Amsterdam: North Holland Publishing Company.

Weber, R. J., \& Castleman, J. 1970. The time it takes to imagine. Perception and Psychophysics, 8, 3, 165-168.

Wehner, T., Vogt, S., \& Stadler, M. 1984. Task-specific EMG characteristics during mental training. Psychological Research, 46, 389-401. 